\title{
Broadening of the Berezinskii-Kosterlitz-Thouless transition by correlated disorder
}

\author{
I. Maccari, L. Benfatto, ${ }^{*}$ and C. Castellani \\ ISC-CNR and Department of Physics, Sapienza University of Rome, P. le A. Moro 5, 00185 Rome, Italy
}

(Received 27 February 2017; revised manuscript received 16 May 2017; published 28 August 2017)

\begin{abstract}
The Berezinskii-Kosterlitz-Thouless (BKT) transition in two-dimensional superconductors is usually expected to be protected against disorder. However, its fingerprints in a real system, such as, e.g., the universal superfluiddensity jump, are often at odds with this expectation. Here, we show that the disorder-induced granularity of the superconducting state modifies the nucleation mechanism for vortex-antivortex pairs. This leads to a considerable smearing of the universal superfluid-density jump as compared to the paradigmatic clean case, in agreement with experimental observations.
\end{abstract}

DOI: 10.1103/PhysRevB.96.060508

More than 40 years after the seminal work by Berezinskii [1], Kosterlitz, and Thouless [2,3], the Berezinskii-KosterlitzThouless (BKT) transition remains one of the most fascinating examples of topological phase transitions. Its universality class describes several phenomena ranging from the quantum metalinsulator transition in one dimension (1D) to the Coulombgas screening transition in $2 \mathrm{D}$, and of course the metal-tosuperfluid transition in 2D [4]. As such, it has been investigated in neutral superfluids, such as, e.g., thin He films [5] and coldatom systems made of bosons [6] or neutral fermions [7], but also in quasi-2D superconductors. The latter case applies not only to thin films of conventional [8-17] and unconventional [18-20] superconductors, but also to the artificially confined $2 \mathrm{D}$ electron gas at the interface between two insulators in artificial heterostructures [21,22], or in the topmost layer of ion-gated superconducting (SC) systems [23].

The most spectacular hallmark of the BKT transition is the expected universal jump of the superfluid density at $T_{\text {BKT }}$ [24]. While this jump has been clearly observed in superfluid helium films [5], in disordered films of conventional superconductors it is systematically smeared out in a broad downturn, as shown by direct measurements of the superfluid density via the inverse penetration depth $\lambda$ [11-17], or by its estimate based on the nonlinear $I-V$ characteristics [8-10]. Since the jump occurs when the superfluid stiffness $J_{s}$ is of the order of $T_{\mathrm{BKT}}$, in conventional superconductors the BKT transition can be clearly distinguished from the BCS one only in thin films. Indeed, by reducing the film thickness $d$, one eliminates the screening effects of supercurrents (making the Pearl [25] length $\Lambda=2 \lambda^{2} / d$ larger than the system size) and suppresses $J_{s}$ by increasing the effective disorder level [26]. This implies that the BKT transition is found in strongly disordered systems, which are usually at the verge of a direct superconductor-to-insulator transition (SIT). As it has been proven experimentally [27-33] and discussed theoretically [37-42] in the last few years, the competition between SC phase coherence and charge localization triggered by strong disorder induces an emergent "granular" SC background, whose effects on the BKT transition have yet to be explored. Interestingly, the inhomogeneity of the SC state can be relevant also for different classes of 2D SC materials, such as

\footnotetext{
*Corresponding author: lara.benfatto@ roma1.infn.it
}

heterostructures [22,34-36], or even ultrathin films of cuprate superconductors [19], where the BKT jump is completely lost in the underdoped phase when superconductivity competes with spin/charge ordering.

Understanding the role of microscopic electronic disorder on the BKT transition within SC fermionic models is an incredible task $[38,40,43,44]$, due mainly to the small size of systems accessible numerically. Alternatively, one can address the question directly within a proper phase-only model [45-48], as, e.g., the $X Y$ model,

$$
H=-\sum_{i j} J_{i j} \cos \left(\theta_{i}-\theta_{j}\right),
$$

where $\theta_{i}$ represents the SC phase and $J_{i j}$ the random Josephson-like coupling between coarse-grained SC islands. As long as the $J_{i j}$ are spatially uncorrelated, the Harris criterium [49] guarantees that disorder is irrelevant at the transition, so that, for example, the expected "universal" jump [24] of the superfluid stiffness at $T_{\mathrm{BKT}}$ is still preserved, as confirmed by numerical simulations [46,47]. In this Rapid Communication we investigate what happens when instead the spatial arrangement of the couplings $J_{i j}$ mimics the granular, spatially correlated disorder observed experimentally, that can be generated by the mean-field solution of the (quantum) $X Y$ model in a random transverse field (RTF) [39,42,50]. While for uncorrelated disorder the robustness of the BKT physics is preserved even away from the transition, with a universal rescaled behavior, the fragmentation of the SC state at strong disorder in the RTF model leads to a pronounced temperature dependence before the transition, with a smoothing of the BKT jump in close analogy with experimental observations [10-17]. This result follows from unconventional vortex-pair nucleation in the granular SC state, made possible by the presence of large clusters of low-SC regions.

The superfluid stiffness $J_{s}$ of the model (1) for a given, let us say $x$, direction is defined as

$$
\begin{aligned}
& J_{s}=J_{d}-J_{p}, \\
& J_{d}=\frac{1}{L^{2}}\left\langle\sum_{i} J_{i, i+x} \cos \left(\theta_{i}-\theta_{i+x}\right)\right\rangle, \\
& J_{p}=\frac{1}{T L^{2}}\left\langle\left(\sum_{i} J_{i, i+x} \sin \left(\theta_{i}-\theta_{i+x}\right)\right)^{2}\right\rangle,
\end{aligned}
$$




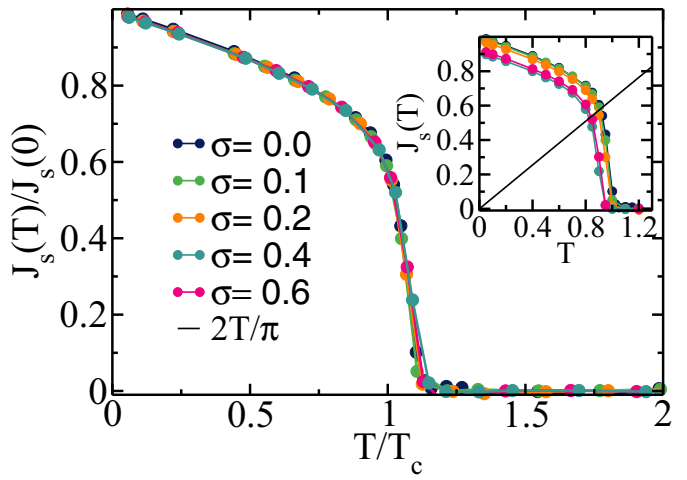

FIG. 1. Superfluid stiffness as a function of the temperature for a Gaussian distribution of the couplings at different values of the variance $\sigma$. In the main panel the curves have been rescaled by the value of the stiffness at $T=0$ and by $T_{c}$, defined as the intersection of $J_{s}(T)$ with the $2 T / \pi$ line, shown in the inset (solid black line).

where $L$ is the size of the square lattice. Here, $J_{d}$ denotes the diamagnetic term, which coincides with the average kinetic energy of the system, while $J_{p}$ is the paramagnetic term, obtained as a current-current correlation function for the paramagnetic current $I_{i, i+x}^{p}=J_{i, i+x} \sin \left(\theta_{i}-\theta_{i+x}\right)$ of the model (1). Equation (2) is computed by means of Monte Carlo simulations on a $L \times L$ square lattice with $L=128$, such that in the clean case $\left(J_{i j}=J\right)$ a sharp jump of $J_{S}$ is recovered (see Ref. [51] for technical details).

To test the effects of uncorrelated disorder we first considered the paradigmatic case where the values of the local couplings $J_{i j}$ are randomly extracted from a Gaussian distribution $P_{G}\left(J_{i j}\right)=\exp \left[-\left(J_{i j}-\bar{J}\right) / 2 \sigma^{2}\right] / \sqrt{2 \pi \sigma^{2}}$ with increasing variance $\sigma$ and fixed average $\bar{J}=1$. The suppression of
$J_{s}(T=0)$ due to disorder is reflected in a suppression of the critical temperature $T_{c}$. The jump of $J_{S}(T)$ occurs at the intersection with the universal $2 T / \pi$ line, so that the universal relation [24] $J_{s}\left(T_{c}\right)=2 T_{c} / \pi$ is always preserved (see the inset of Fig. 1). This is in agreement with the Harris criterium [49]: Since at the BKT transition the correlation length diverges exponentially [3] instead of the usual power law, the length scale set by disorder is always irrelevant for the ordering process at criticality. However, by rescaling the $J_{s}(T)$ curves by their $T=0$ value and the temperature by $T_{c}$ we find a remarkable collapse of all the curves on each other, showing the complete irrelevance of disorder even away from $T_{c}$. This behavior can be understood considering that at low temperature the primary excitations of the model (1) are disordered longitudinal spin waves, well described [52] by the quadratic approximation of the Hamiltonian (1), $H \approx \int d \mathbf{r} J(\mathbf{r})[\nabla \theta(\mathbf{r})]^{2}$. By making an expansion of the local stiffness $J(\mathbf{r})=\bar{J}+\delta J(\mathbf{r})$ around its average value $\bar{J}$, one can show [52] that at low temperatures,

$$
J_{d} \simeq \bar{J}-T / 4, \quad J_{p}=\bar{J}\left[\left\langle\delta J^{2}\right\rangle / 2 \bar{J}^{2}+c(T / \bar{J})^{2}\right],
$$

where $c$ is a numerical constant. As a consequence, the primary effect of disorder is to induce a $T=0$ paramagnetic suppression of the stiffness $J_{s}^{\text {app }}(T=0) \simeq \bar{J}\left[1-\left\langle\delta J^{2}\right\rangle / 2 \bar{J}^{2}\right]$, as found [45] also by using the mapping [53] into a randomresistor network with conductance $J_{i j}$ at each node. On the other hand, the $-T / 4$ spin-wave depletion of the diamagnetic term is unaffected by disorder, so that the $T_{c}$, determined by the universal relation, follows from $T_{c} \simeq(\pi / 2)\left[J_{s}(0)-T_{c} / 4\right]$, i.e., it scales itself with $J_{s}(0)$. This explains why the rescaled curves of Fig. 1 collapse on each other.

Let us consider now the case of correlated disorder, relevant for thin SC films. Tunneling spectroscopy on several conventional superconductors such as $\mathrm{NbN}, \mathrm{InO}_{x}$, and $\mathrm{TiN}$

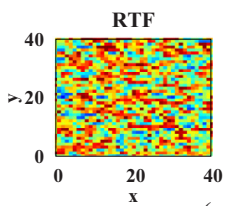

(a1)

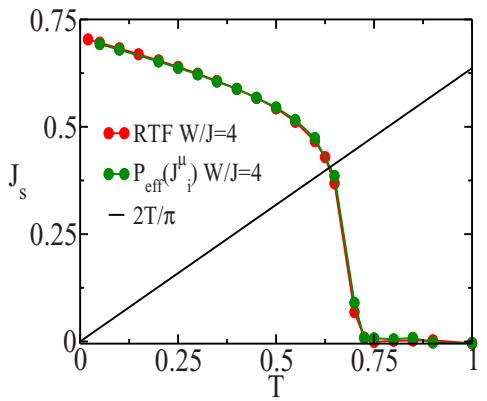

(a2)

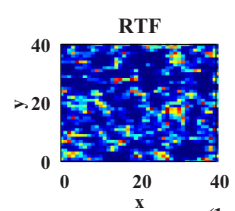

(b1)
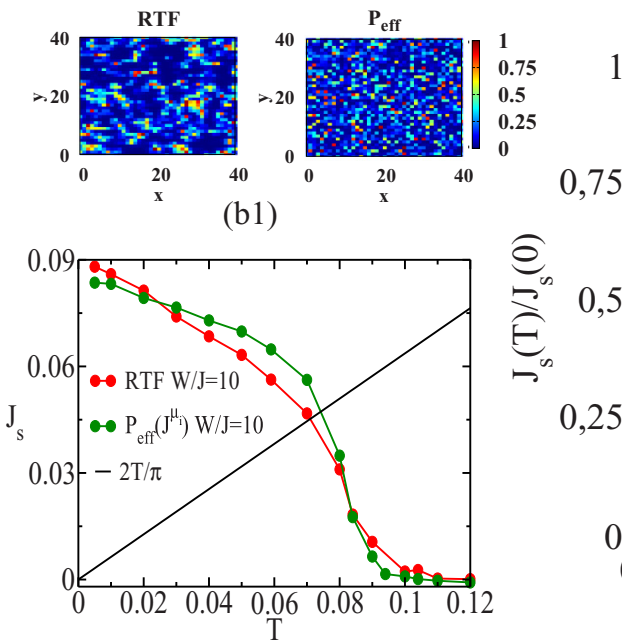

(b2)

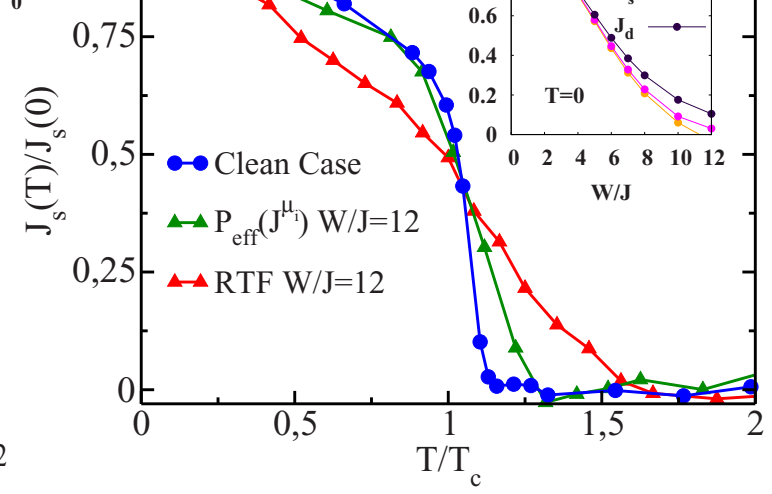

(c)

FIG. 2. Maps of the couplings $J_{i, i+x}[(\mathrm{a} 1),(\mathrm{b} 1)]$ and superfluid stiffness [(a2), (b2)] at disorder levels $W / J=4$ and $W / J=10$. Here, RTF and $P_{\text {eff }}$ denote the case of correlated and uncorrelated disorder, respectively. At weaker disorder (a2) the two curves overlap and the jump of $J_{s}$ is still sharp. At larger disorder (b2) the curve for the RTF case starts to deviate from the usual trend, showing an almost symmetric smearing of the jump around the critical temperature. (c) Rescaled curves of the superfluid stiffness for the clean case, the uncorrelated $P_{\text {eff }}$, and correlated RTF disordered case at $W / J=12$. Despite strong disorder, the $P_{\text {eff }}$ curve shows only a small finite-size effect above $T_{c}$, while the RTF stiffness is dramatically modified above and below the transition. Inset: Evolution with disorder of the zero-temperature value of $J_{s}$, of $J_{d}$ for the RTF model, and of the approximate result $J_{s}^{\text {app }}$ obtained from (5). 


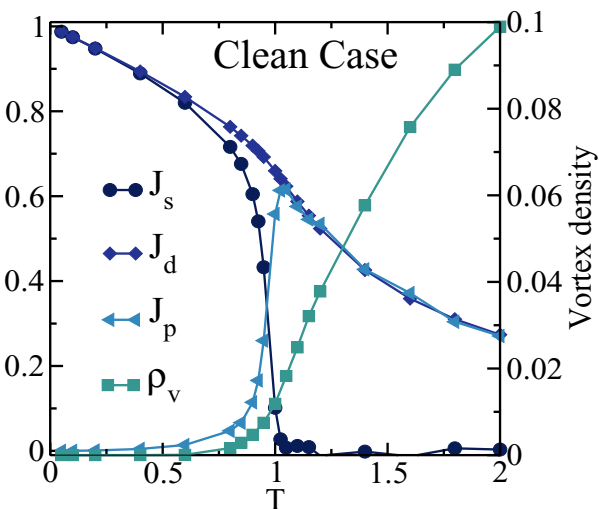

(a)

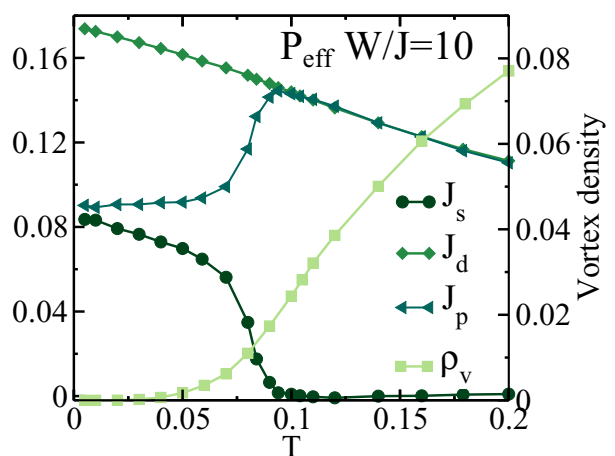

(b)

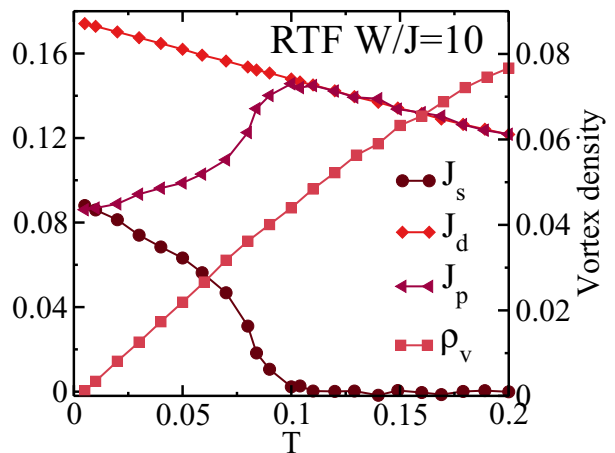

(c)

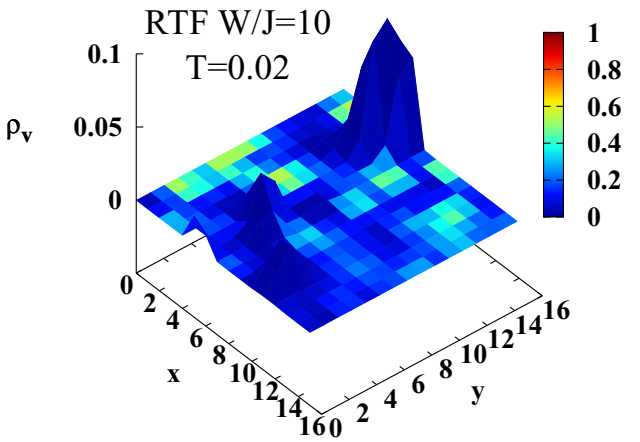

(d)

FIG. 3. Temperature dependence of the superfluid stiffness $J_{s}$, the diamagnetic term $J_{d}$, the paramagnetic term $J_{p}$, and the vortex-pair density $\rho_{v}$ for three different cases: (a) Clean case, (b) uncorrelated disorder $P_{\text {eff }}$ with $W / J=10$, and (c) correlated disorder RTF with $W / J=10$. (d) Local vortex density $\rho_{V}$ at $T=0.02$ superimposed on the color map of the local stiffness $J_{i j}$ for the RTF model. has shown that the local order parameter, as probed by the local density of states, is strongly inhomogeneous, forming domains of good and bad SC regions with a size of a few times the coherence length $[27,29,33,42]$. This emergent granularity can be reproduced $[39,42,50]$ by the effective quantum $X Y$ pseudospin- $\frac{1}{2}$ model in a transverse random field [54],

$$
\mathcal{H}_{\mathrm{PS}} \equiv-2 \sum_{i} \xi_{i} S_{i}^{z}-2 J \sum_{\langle i, j\rangle}\left(S_{i}^{+} S_{j}^{-}+\text {H.c. }\right) .
$$

Within the bosonic picture of the model (6) the pairing degrees of freedom are frozen, and disorder induces a direct SIT, as seen experimentally [33]. In pseuodospin language, superconductivity corresponds to a spontaneous in-plane magnetization, favored by the coupling $J$, while the random transverse field $\xi_{i}$, box distributed between $-W$ and $W$, tends to align the local spin along $z$, mimicking the localization of Cooper pairs due to disorder. At the mean-field level one easily finds [51] that the local magnetization forms an angle $\phi_{i}^{\mathrm{ps}}$ with respect to the $z$ axis, with $\phi_{i}^{\mathrm{ps}}$ approaching 0 as $W / J$ increases. The resulting maps of the local SC order parameter $\left\langle S_{i}^{x}\right\rangle=\frac{1}{2} \sin \phi_{i}^{\mathrm{ps}}$ reproduce the salient features of the experiments, as discussed previously [27,42]. Here, we investigate the effects of classical phase fluctuations on top of this inhomogeneous SC ground state, described [50,51] by the model (1) with local stiffness $J_{i j}=J \sin \phi_{i}^{\mathrm{ps}} \sin \phi_{j}^{\mathrm{ps}}$. The typical maps of local couplings are shown in the insets of Figs. 2(a1) and 2(b1). As discussed in Refs. [42,50], the local stiffness $J_{i j}$ is on average suppressed by disorder, and it tends to segregate in bad SC regions embedding a good, filamentary [41] SC structure. To disentangle the effects of the spatial correlations of the couplings from the ones connected to their probability distribution, we also compute for each disorder level the stiffness of the effective, uncorrelated distribution $P_{\text {eff. }}$ This means that we assign the value $J_{i j}$ to each link by extracting it randomly from the same probability distribution $P_{\text {eff }}\left(J_{i j}\right)$ which represents the RTF maps. In this case, the SC state does not show any evident aggregation in real space, giving rise to standard, uncorrelated disorder, as it is already evident in the maps shown in Figs. 2(a1) and 2(b1). To quantify these differences we computed [51] for each type of disorder the average size of bad and good regions, identified by a threshold $\alpha J_{s}$, with $\alpha \leqslant 1$. Looking, e.g., to the bad clusters, one sees that while for the $P_{\text {eff }}$ their size is almost independent on $W / J$ and $\alpha$, for the RTF maps it increases significantly with $W / J$ and $\alpha$, showing a clear tendency towards an emergent clusterization of the SC state.

The evolution of the stiffness computed by means of Monte Carlo simulations for increasing disorder level $W / J$ (with $J=$ 1 ) is shown in Fig. 2. The $T=0$ suppression of the stiffness is well captured by the approximated expression (5) up to large $W / J$ values [see the inset of Fig. 2(c)]. With respect to the Gaussian case discussed above, here the diamagnetic term is rapidly suppressed for increasing $W / J$. On the other hand, up to $W / J=4$ the BKT transition preserves its character, and spatial correlations are irrelevant, as shown in Fig. 2(a2). However, at larger disorder the granularity of the SC state increases, and the superfluid-density jumps start to be smeared out [see Fig. 2(b2)]. Despite this, the same effect is not seen when spatial correlations disappear, as demonstrated by the 
case of $P_{\text {eff. }}$. To compare the behavior at different disorder levels we show in Fig. 2(c) the rescaled curves. At $W / J=12$ the probability distribution of the coupling is peaked at low values with very large tails $[39,42,51]$. This has the only effect to increase slightly the finite-size effect, as one can see by comparing the curve for $P_{\text {eff }}$ with the clean case. However, the granular RTF model shows a definitively broader jump, which is symmetrically smeared out around $T_{c}$, in agreement with experimental observations for the inverse penetration depth [11-17] or for the nonlinear $I-V$ exponent [8-10], that we show explicitly in Ref. [51].

To get deeper insight on the separate roles of spin-wave and vortex excitations, we show in Fig. 3 the temperature evolution of the two separate diamagnetic (3) and paramagnetic (4) contributions, along with the average density $\rho_{V}$ of vortex pairs. This is defined by computing the local (positive or negative) vorticity of the phase around each square plaquette of the array. In Fig. 3(a) we show the results for the clean case. As discussed in Eq. (5) above, spin waves dominate the behavior of $J_{d}$ and $J_{p}$ at low temperatures. The vortex density is exponentially suppressed at low $T$ and it increases sharply at $T \simeq 0.9$, bringing up the paramagnetic contribution, which grows quickly, leading to $J_{p}=J_{d}$ at $T \simeq 1$. A similar trend is observed at $W / J=10$ for the $P_{\text {eff }}$ case [see Fig. 3(b)]. Indeed, apart from the sizable finite corrections (5) to $J_{d}$ and $J_{p}$ at $T=0$, the thermal evolution of the various contributions is essentially the same: The vortex density has a fast increase only at $T \simeq 0.075$, where the universal jump is indeed expected [see Fig. 2(b)]. Instead, the results change considerably for the RTF model [Fig. 3(c)]. In particular, we observe an anomalously smooth increase of the paramagnetic response $J_{p}$ at low temperature, followed by a faster one around the temperature scale where the universal jump should be observed. This unconventional paramagnetic response is responsible for the symmetric broadening of the transition observed in Fig. 2(b). A second striking result is the almost linear increase of the vortex density in the whole temperature range.

To get deeper insight into the anomalous behavior of $J_{p}$ at strong disorder, we investigated the vortex nucleation mechanism in real space. As we explained above, a distinct characteristic of the RTF model is the emergence of large clusters of bad SC regions, where the local stiffness is small [51]. Vortices can then proliferate inside these regions already at low temperatures, as shown in Fig. 3(d), where the local vorticity is superimposed on the color map of the local stiffness. In contrast, in the $P_{\text {eff }}$ case the bad regions remain small, and vortex formation cannot be confined in low-coupling clusters. The direct connection between $J_{p}$ and $\rho_{V}$ in the RTF model is also proven by adding explicitly to the model (1) a chemical-potential term weighting the finite vortex density [51]. While for the homogeneous case the only effect this term has is to shift the transition to higher temperatures [55], for the RTF case it strongly modifies both $J_{p}$ and $\rho_{V}$ at low temperatures, showing that for correlated disorder, vortex-antivortex pairs are relevant already below $T_{c}$, in contrast to what was shown in Fig. 1 for uncorrelated disorder. Finally, we find that these anomalies are observed by increasing the lattice size up to $L=256$ [51], suggesting that the effects observed here are very different from the usual rounding of the stiffness above $T_{c}$ due to the conventional finite-size effect.

In summary, we investigated by Monte Carlo simulations the evolution of the universal superfluid-density jump within a $X Y$ model with random local couplings. We compared models with and without spatial correlations, focusing on the temperature dependence of the superfluid stiffness. When disorder lacks spatial structure, it appears irrelevant not only for the jump at criticality, as expected, but also away from it. Indeed, by rescaling the stiffness to its $T=0$ value, suppressed by disorder, we observe a remarkable universal temperature dependence. This scenario changes considerably when disorder mimics the inhomogeneous SC background observed experimentally. In this case the superfluid-density jump is considerably smeared out both above and below the temperature where the universal jump would be expected. This effect is attributed to a different mechanism for vortex-antivortex pair generation due to the presence of large clusters of low-SC regions. Our results not only provide an explanation for the trends observed experimentally in thin films of conventional [8-17] and unconventional [19] superconductors, but they pave the way for the understanding of the topological excitations in gated 2D superconductors, where the inhomogeneity of the SC state is recently emerging [22,34-36] as the hallmark of field-induced electron doping.

We thank J. Lorenzana for useful discussions. This work has been supported by MIUR under Project No. PRINRIDEIRON-2012X3YFZ2 and by MAECI under the ItalianIndia Collaborative Project No. SUPERTOP-PGR04879.
[1] V. L. Berezinskii, Sov. Phys. JETP 34, 610 (1972).

[2] J. M. Kosterlitz and D. J. Thouless, J. Phys. C 6, 1181 (1973).

[3] J. M. Kosterlitz, J. Phys. C 7, 1046 (1974).

[4] For a recent review, see 40 Years of Berezinskii-KosterlitzThouless Theory, edited by J. V. José (World Scientific, Singapore, 2013).

[5] D. McQueeney, G. Agnolet, and J. D. Reppy, Phys. Rev. Lett. 52, 1325 (1984).

[6] Z. Hadzibabic, P. Krüger, M. Cheneau, B. Battelier, and J. Dalibard, Nature (London) 441, 1118 (2006).
[7] P. A. Murthy, I. Boettcher, L. Bayha, M. Holzmann, D. Kedar, M. Neidig, M. G. Ries, A. N. Wenz, G. Zürn, and S. Jochim, Phys. Rev. Lett. 115, 010401 (2015).

[8] K. Epstein, A. M. Goldman, and A. M. Kadin, Phys. Rev. Lett. 47, 534 (1981).

[9] A. M. Kadin, K. Epstein, and A. M. Goldman, Phys. Rev. B 27, 6691 (1983).

[10] A. T. Fiory, A. F. Hebard, and W. I. Glaberson, Phys. Rev. B 28, 5075 (1983).

[11] S. J. Turneaure, T. R. Lemberger, and J. M. Graybeal, Phys. Rev. Lett. 84, 987 (2000). 
[12] R. W. Crane, N. P. Armitage, A. Johansson, G. Sambandamurthy, D. Shahar, and G. Gruner, Phys. Rev. B 75, 094506 (2007).

[13] W. Liu, M. Kim, G. Sambandamurthy, and N. P. Armitage, Phys. Rev. B 84, 024511 (2011).

[14] A. Kamlapure, M. Mondal, M. Chand, A. Mishra, J. Jesudasan, V. Bagwe, L. Benfatto, V. Tripathi, and P. Raychaudhuri, Appl. Phys. Lett. 96, 072509 (2010).

[15] M. Mondal, S. Kumar, M. Chand, A. Kamlapure, G. Saraswat, G. Seibold, L. Benfatto, and P. Raychaudhuri, Phys. Rev. Lett. 107, 217003 (2011).

[16] S. Misra, L. Urban, M. Kim, G. Sambandamurthy, and A. Yazdani, Phys. Rev. Lett. 110, 037002 (2013).

[17] J. Yong, T. R. Lemberger, L. Benfatto, K. Ilin, and M. Siegel, Phys. Rev. B 87, 184505 (2013).

[18] I. Hetel, T. R. Lemberger, and M. Randeria, Nat. Phys. 3, 700 (2007).

[19] J. Yong, M. J. Hinton, A. McCray, M. Randeria, M. Naamneh, A. Kanigel, and T. R. Lemberger, Phys. Rev. B 85, 180507 (2012), and references therein.

[20] P. G. Baity, X. Shi, Z. Shi, L. Benfatto, and D. Popović, Phys. Rev. B 93, 024519 (2016).

[21] J. A. Bert, K. C. Nowack, B. Kalisky, H. Noad, J. R. Kirtley, C. Bell, H. K. Sato, M. Hosoda, Y. Hikita, H. Y. Hwang, and K. A. Moler, Phys. Rev. B 86, 060503(R) (2012).

[22] G. N. Daptary, S. Kumar, P. Kumar, A. Dogra, N. Mohanta, A. Taraphder, and A. Bid, Phys. Rev. B 94, 085104 (2016).

[23] Y. Saito, Y. Kasahara, J. Ye, Y. Iwasa, and T. Nojima, Science 350, 409 (2015).

[24] D. R. Nelson and J. M. Kosterlitz, Phys. Rev. Lett. 39, 1201 (1977).

[25] J. Pearl, Appl. Phys. Lett. 5, 65 (1964).

[26] M. R. Beasley, J. E. Mooij, and T. P. Orlando, Phys. Rev. Lett. 42, 1165 (1979).

[27] B. Sacepe, C. Chapelier, T. I. Baturina, V. M. Vinokur, M. R. Baklanov, and M. Sanquer, Nat. Commun. 1, 140 (2010); B. Sacépé et al., Nat. Phys. 7, 239 (2011).

[28] M. Mondal, A. Kamlapure, M. Chand, G. Saraswat, S. Kumar, J. Jesudasan, L. Benfatto, V. Tripathi, and P. Raychaudhuri, Phys. Rev. Lett. 106, 047001 (2011).

[29] A. Kamlapure, T. Das, S. Chandra Ganguli, J. B. Parmar, S. Bhattacharyya, and P. Raychaudhuri, Sci. Rep. 3, 2979 (2013).

[30] Y. Noat, V. Cherkez, C. Brun, T. Cren, C. Carbillet, F. Debontridder, K. Ilin, M. Siegel, A. Semenov, H.-W. Hübers, and D. Roditchev, Phys. Rev. B 88, 014503 (2013).

[31] C. Brun, T. Cren, V. Cherkez, F. Debontridder, S. Pons, D. Fokin, M. C. Tringides, S. Bozhko, L. B. Ioffe, B. L. Altshuler, and D. Roditchev, Nat. Phys. 10, 444 (2014).

[32] C. Carbillet, S. Caprara, M. Grilli, C. Brun, T. Cren, F. Debontridder, B. Vignolle, W. Tabis, D. Demaille, L. Largeau,
K. Ilin, M. Siegel, D. Roditchev, and B. Leridon, Phys. Rev. B 93, 144509 (2016)

[33] C. Brun, T. Cren, and D. Roditchev, Supercond. Sci. Technol. 30, 013003 (2017).

[34] J. Biscaras, N. Bergeal, S. Hurand, C. Feuillet-Palma, A. Rastogi, R. C. Budhani, M. Grilli, S. Caprara, and J. Lesueur, Nat. Mater. 12, 542 (2013).

[35] G. E. D. K. Prawiroatmodjo, F. Trier, D. V. Christensen, Y. Chen, N. Pryds, and T. S. Jespersen, Phys. Rev. B 93, 184504 (2016).

[36] G. Singh, A. Jouan, L. Benfatto, F. Couedo, P. Kumar, A. Dogra, R. Budhani, S. Caprara, M. Grilli, E. Lesne, A. Barthelemy, M. Bibes, C. Feuillet-Palma, J. Lesueur, and N. Bergeal, arXiv:1704.03365.

[37] A. Ghosal, M. Randeria, and N. Trivedi, Phys. Rev. B 65, 014501 (2001).

[38] Y. Dubi, Y. Meir, and Y. Avishai, Nature (London) 449, 876 (2007).

[39] L. B. Ioffe and M. Mezard, Phys. Rev. Lett. 105, 037001 (2010); M. V. Feigelman, L. B. Ioffe, and M. Mezard, Phys. Rev. B 82, 184534 (2010).

[40] K. Bouadim, Y. L. Loh, M. Randeria, and N. Trivedi, Nat. Phys. 7, 884 (2011)

[41] G. Seibold, L. Benfatto, C. Castellani, and J. Lorenzana, Phys. Rev. Lett. 108, 207004 (2012).

[42] G. Lemarié, A. Kamlapure, D. Bucheli, L. Benfatto, J. Lorenzana, G. Seibold, S. C. Ganguli, P. Raychaudhuri, and C. Castellani, Phys. Rev. B 87, 184509 (2013).

[43] A. Erez and Y. Meir, Europhys. Lett. 91, 47003 (2010).

[44] E. J. König, A. Levchenko, I. V. Protopopov, I. V. Gornyi, I. S. Burmistrov, and A. D. Mirlin, Phys. Rev. B 92, 214503 (2015).

[45] S. Barabash, D. Stroud, and I.-J. Hwang, Phys. Rev. B 61, R14924 (2000)

[46] G. M. Wysin, A. R. Pereira, I. A. Marques, S. A. Leonel, and P. Z. Coura, Phys. Rev. B 72, 094418 (2005).

[47] A. Erez and Y. Meir, Phys. Rev. Lett. 111, 187002 (2013).

[48] M. Swanson, Y.-L. Loh, M. Randeria, and N. Trivedi, Phys. Rev. X 4, 021007 (2014).

[49] A. B. Harris, J. Phys. C 7, 1671 (1974).

[50] T. Cea, D. Bucheli, G. Seibold, L. Benfatto, J. Lorenzana, and C. Castellani, Phys. Rev. B 89, 174506 (2014).

[51] See Supplemental Material at http://link.aps.org/supplemental/ 10.1103/PhysRevB.96.060508 for technical details on the Monte Carlo simulations, for additional information on the disorder characterisation for the RTF model and for a discussion of the $I-V$ characteristics.

[52] I. Maccari, L. Benfatto, and C. Castellani (unpublished).

[53] S. Kirkpatrick, Rev. Mod. Phys. 45, 574 (1973).

[54] M. Ma and P. A. Lee, Phys. Rev. B 32, 5658 (1985).

[55] L. Benfatto, C. Castellani, and T. Giamarchi, Phys. Rev. Lett. 98, 117008 (2007) 